\title{
Image Enlargement Based on the Different Scale Factors for Slice Region
}

\author{
I Komang Somawirata ${ }^{\text {a, }}$, Keiichi Uchimura ${ }^{\text {a }}$, Gou Koutaki ${ }^{\text {a }}$ \\ ${ }^{a}$ Computer Science and Electrical Engineering \\ Graduate School of Science and Technology, Kumamoto University, Japan \\ *Corresponding Author: kmgsomawirata@navi.cs.kumamoto-u.ac.jp
}

\begin{abstract}
This paper proposes an image enlargement method with proportional content magnification by implementing the different scale factor for image slice region. We use window-kernel image magnification to enlarge the image slice. The enlarged image to target image size has three steps. The first step, source image is enlarged to the same size with high of the target image. The second step, the enlarged image in the first step is sliced from top to bottom following the minimum energy from the image. The third step, the image slices with non salient image content are selected to enlarge to the full-size image. The proposed method has been evaluated using peak signal-to-noise ratio (PSNR). The PSNR value on a uniform enlarged image using a scale factor equal to four has highest value compared with the comparison method. The image enlargement in the different ratio is also presented.
\end{abstract}

Keywords: enlargement, window, slice.

\section{Introduction}

Image enlargement is to change the smaller image size to the larger image size. Generally, the image enlargement using interpolated pixels are resulted in the uniform image resizing. Currently, the various sizes and ratios of displays have been presented. The ratios of display size are ranging from 3:2, 4:3, 5:4, 16:9, 16:10 and 17:9 respectively for the width and height.

The classic problem in image enlargement is the image look blurred and jagged in edge section. Another problem of image enlargement is non-proportional content on the image enlargement in different ratios.

The image enlargement methods have been developed to improve the quality of image enlargement result, such as the image enlargement based on regularity on the geometric $^{(1)}$. This method is called by new edge-directed interpolation (NEDI). NEDI method uses covariance-based adaptive interpolation especially in edge and pixels near of edge, for non edge pixels they use bilinear interpolation. This method is uniform image enlargement and based on the two-times image magnification. NEDI method is difficult to implement on image enlargement in different ratios. The recovery of sharp edges on HR image uses reverse diffusion interpolation (RDI) ${ }^{(2)}$ is capable to reduce high frequency in edge image. Reverse diffusion can be considered as a nonlinear high-pass filter. However, RDI method is only for uniform image enlargement. Image enlargement uses weighting technique to produce a better HR image is implemented on Weighted Sum of Linear Extrapolations $(\mathrm{WLE})^{(3)}$. The WLE method explains the interpolation in three different positions: interpolation between two horizontal pixels, interpolation between two vertical pixels, and interpolation between four pixels. However, WLE method does not provide a good result, especially for enlarging an image on scale factor greater than two and this methods only for uniform image enlargement.

Another image resizing, which implement the image analysis to obtain the important region ${ }^{(4,5)}$. These methods are implemented only for small screen size. Many retargeting methods have been proposed to resize the image, especially for down scale and maintain salient content in the image ${ }^{(6,7,8)}$. However, the image enlargement (up scaling) in the different ratio is not presented.

Therefore, the purpose of this study is to obtain a good quality of image enlargement and its results an enlarged image with proportional content in different ratios.

\section{Proposed Method}

This research proposes a method of image enlargement by a combination between window - kernel 


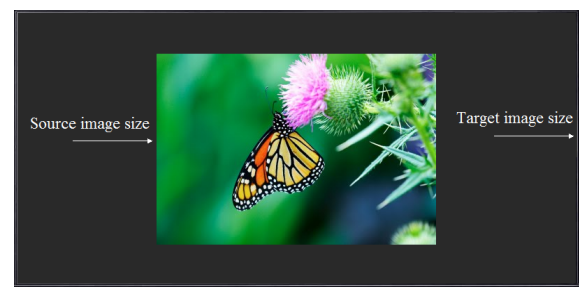

(a)

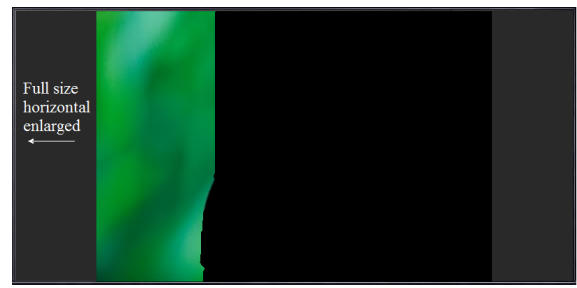

(d)

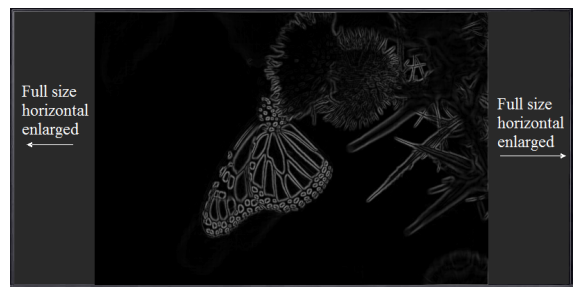

(b)

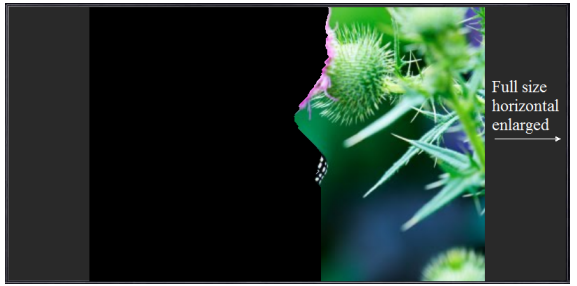

(e)

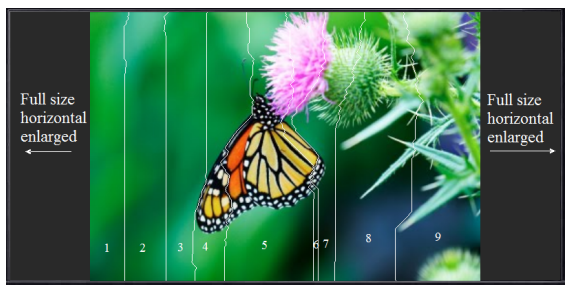

(c)

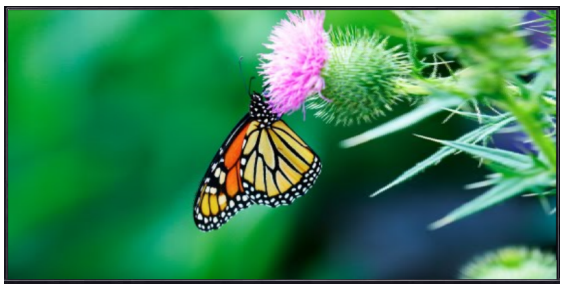

(f)

Fig.1. The steps of image enlargement. (a) Target image and source image sizes, (b) Energy of image, (c) Top to bottom image slicing based on the minimum energy of image, (d) and (e) are left and right image selection, (f) The result of image enlargement.

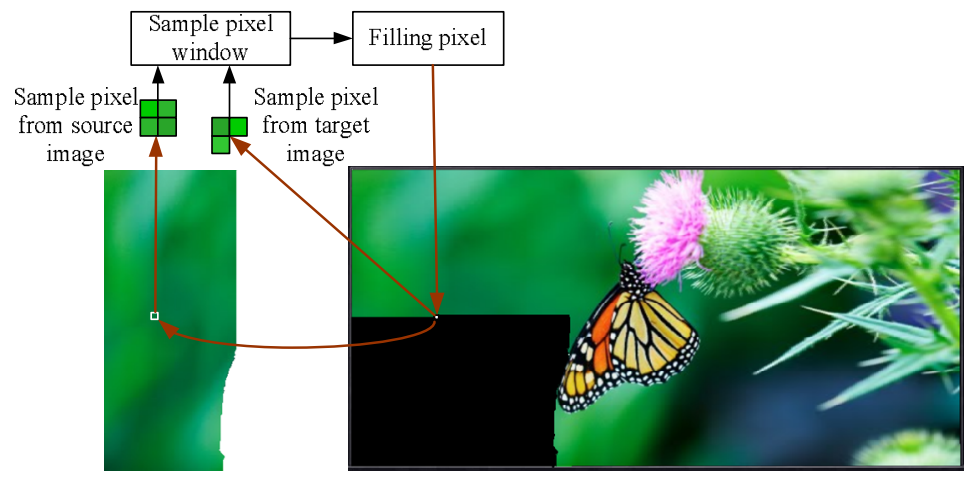

Fig. 2. Image enlargement based on the filling pixel using window-kernel

scaling method and slicing method. Figure 1 shows the process of image enlargement. The source and target image sizes are in the different ratio, such as shown in Fig.1.(a). The first step, source image is enlarged to the same size with high of the target image $\left(\hat{f}_{o}\right)$. The second step, the image enlargement in the first step is sliced from top to bottom following the minimum energy in image. The image energy and slice image are shown in Figs.1.(b) and (c), respectively. The number of image slice in Fig.1.(c) is nine. The third step is selecting the slice image to be enlarged. Figs.1.(d) and (e) show the left and right image slice selection. The enlargement image result is shown in Fig.1.(f).

\subsection{Image Enlargement Based on the Filling Pixel}

The image enlargement process is illustrated as in Fig.2, which is based on filling pixel. The steps of image enlargement based on the filling pixel are presented as follows:

- The first step is finding the corresponding coordinates in the source image for the sample pixel kernel. The corresponding coordinate is obtained by dividing the height $\left(n_{1}\right)$ and width $\left(n_{2}\right)$ coordinates of the target image with the scale factors $\left(S_{h}\right.$ and $\left.S_{w}\right)$, as shown in Eq. (1).

$c_{h}=\frac{n_{1}}{S_{h}}$ and $c_{w}=\frac{n_{2}}{S_{w}}$

- The second step is taking four pixels for the sample pixel window.

- The third step is calculating one pixel for filling the corresponding coordinate in the target image size.

\section{(a) Sample Pixel Window}

The sample pixel window contains four pixels, which is placed in the $2 \times 2$ window size. The fourth sample pixel is obtained from the source image $f(x, y)$ and the target image 
$f_{T}\left(n_{1}, n_{2}\right)$ which has been filled by pixel. Equation (1) represents the relationship coordinate between the source image and target image. Referring to Eq. (1), there are three rules to fill the sample pixel window $\left(w_{i j}^{s}\right)$.

- If the value of $c_{h}$ is fractional and the $c_{w}$ is an integer, then the element of the sample pixel window is as in equation (2).

$$
w_{i j}^{s}=\left|\begin{array}{cc}
f(x, y) & f_{T}\left(n_{1}-1, n_{2}\right) \\
f(x+1, y) & f_{T}\left(n_{1}, n_{2}-1\right)
\end{array}\right|
$$

- If the value of $c_{h}$ is integer and the $c_{w}$ is an fractional, then the element of the sample pixel window is as in equation (3).

$$
w_{i j}^{s}=\left|\begin{array}{cc}
f(x, y) & f(x, y+1) \\
f_{T}\left(n_{1}-1, n_{2}-1\right) & f_{T}\left(n_{1}-1, n_{2}\right)
\end{array}\right|
$$

- If the value of $c_{h}$ is fractional and the $c_{w}$ is an fractional, then the element of the sample pixel window is as in equation (4).

$$
w_{i j}^{s}=\left|\begin{array}{cc}
f(x, y) & f(x, y+1) \\
f(x+1, y) & f(x+1, y+1)
\end{array}\right|
$$

(b) Weight window

The weight window has the same size matrix with a sample pixel. Weight window is symbolized by $w_{i j}^{w}$.

Each coordinate $n_{1}$ and $n_{2}$ have two weights. The coordinate $n_{1}$ has weights $w_{x i}$, where $i=1,2$. As for the $n_{2}$ coordinates have weights $w_{y i}$. Matrix element of $w_{i j}^{w}$ values is calculated by multiplying the value in each coordinate $w_{x i}$ with $w_{y i}$.

$$
\begin{aligned}
& w_{i j}^{w}=\left|\begin{array}{ll}
w_{11} & w_{12} \\
w_{21} & w_{22}
\end{array}\right| \\
& w_{x 1}=\left\lceil c_{h}\right\rceil-c_{h} \text { and } w_{x 2}=1-w_{x 1} \\
& w_{y 1}=\left\lceil c_{w}\right\rceil-c_{w} \text { and } w_{y 2}=1-w_{y 1}
\end{aligned}
$$

Wherein, $w_{x i}$ and $w_{y j}$ are weight value for the height and width. $w_{i j}$ is the element matrix of weight window $\left(w_{i j}=w_{x i} w_{y j}\right.$ and $i=j=1,2)$.

\section{(c) Filling pixel}

A pixel for fill the corresponding coordinate in target image is obtained by multiplying the sample pixel window with weight window as shown in Eq. (8). $\widehat{f}\left(n_{1}, n_{2}\right)=\sum_{i=1}^{2} \sum_{j=1}^{2} w_{i j}^{s} w_{i j}^{w}$

\subsection{The Slice Image Method}

The image slice is based on energy in the image. Calculation of the energy and image slicing process is as follows:

(a) The Energy of Image

We calculate the energy of image using Sobel kernel. There are two Sobel kernel such as in Eqs.(9) and (10). The variable $P$ is the setting power of energy. From the experimental results, the minimum value of $P$ is $0.25 . E$ is two images energy ( $E_{h}$ and $E_{v}$ ) which each point contains the horizontal and vertical absolute derivative approximations, the computations are as in Eq. (11).

$$
\begin{aligned}
& H_{h}=P\left|\begin{array}{ccc}
1 & 0 & -1 \\
2 & 0 & -2 \\
1 & 0 & -1
\end{array}\right| \\
& H_{v}=P\left|\begin{array}{ccc}
1 & 2 & 1 \\
0 & 0 & 0 \\
-1 & -2 & -1
\end{array}\right| \\
& E=\frac{1}{2}\left|\hat{f}_{o} * H_{h}\right|+\frac{1}{2}\left|\hat{f}_{o} * H_{v}\right|
\end{aligned}
$$

Where $E$ is the energy of the image $\hat{f}_{o}$ as in Fig.1.(b). $\hat{f}_{o}$ is image enlargement in the first step. $H_{h}$ and $H_{v}$ is horizontal and vertical derivative filters.

(b) Image Slicing Based on the Energy of Image

Image slicing is done from top to bottom following the minimum-energy. We connect the minimum-energy and mark it with a maximum intensity of pixels (255) as shown in Fig.1. (c). Slicing starts at every distance Slice $_{k}$ as many $m_{s}$ as shown in Eqs.(12) and (13).

$$
\begin{aligned}
& L=\frac{\text { width }}{m_{s}} \\
& \text { Slice }_{k}=\text { Slice }_{k-1}+L
\end{aligned}
$$

Wherein, $L$ is the distance between start point. windth is the width of image enalrgement in the first step $\left(\hat{f}_{o}\right) . m_{s}$ is the maximum number of slice image. Slice $_{k}$ is starting point of image slicing, with $k=1,2,3, \ldots, m_{s}$.

\section{Experimental Result}

In this experiment, we use the standard image such as Lena, Peppers, Baboon and Clown to test our method in uniform image enlargement. Other images such as 


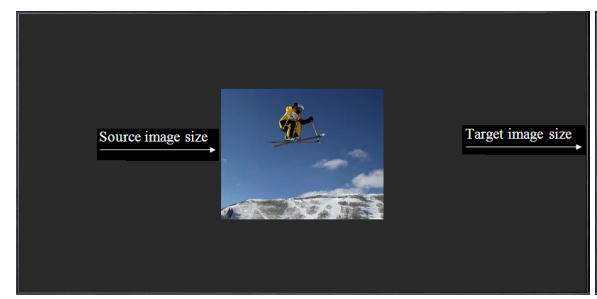

(a)

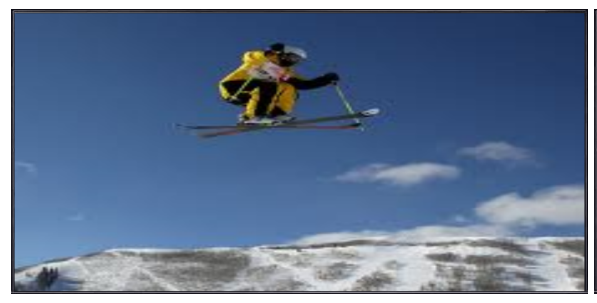

(d)

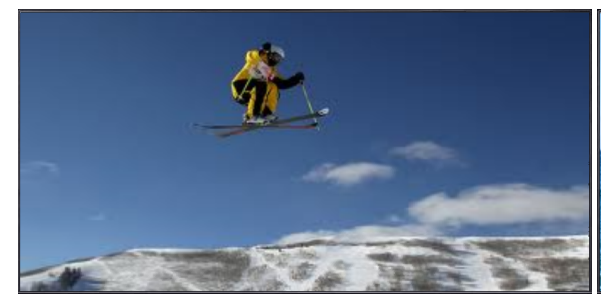

(g)

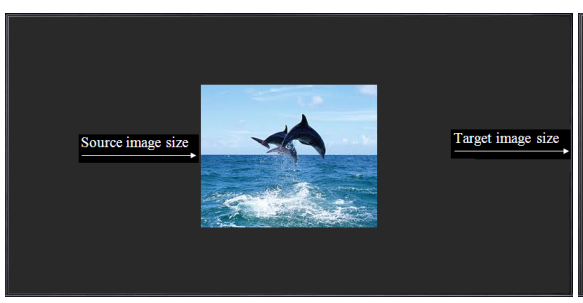

(b)

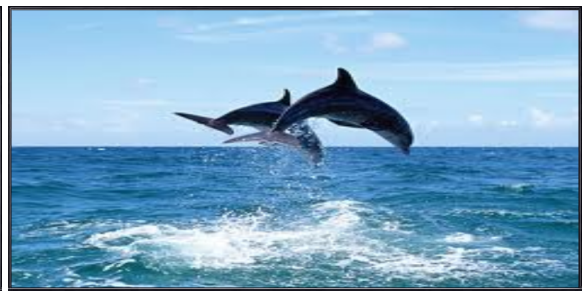

(e)

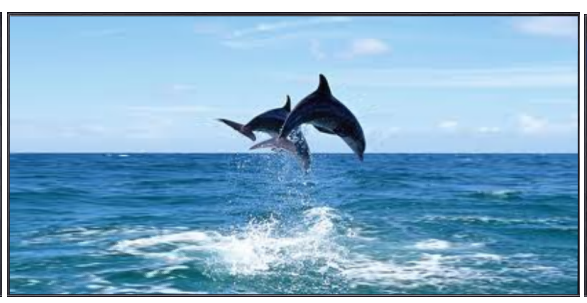

(h)

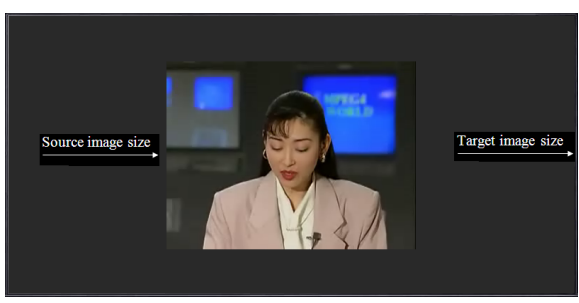

(c)

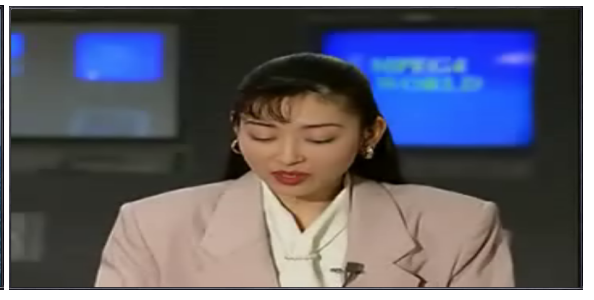

(f)

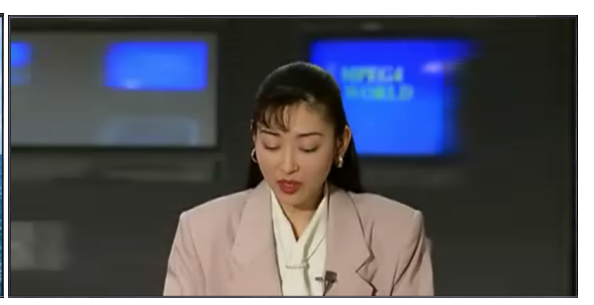

(i)

Fig.3. Target image size with different ratio. (a),(b) and (c) Source image size and target image size. (d), (e) and (f) are the result of enlarged image using uniform scaling method. $(\mathrm{g})$, (h) and (i) are the image enlargement using proposed method.

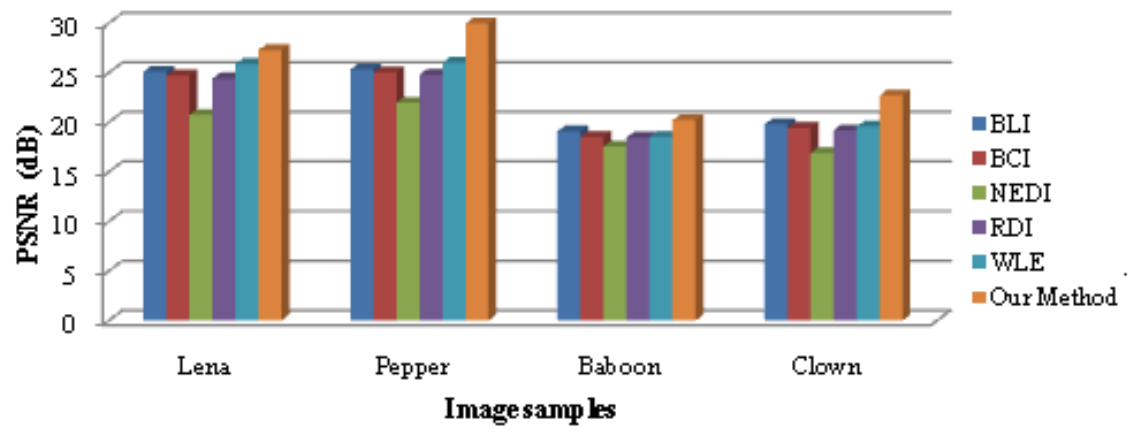

Fig.4. The comparison of PSNR value on uniform image enlargemet using scale factor equal to four.

ski-Jumping, Dolphin and Akiyo is used to image enlargement in different ratios.

\subsection{Qualitative Evaluation}

Figure 3 shows an image enlargement in different ratios. Figures 3 (a), (b) and (c) is the target image size and image source for Ski-jumping, Dolphin and Akiyo, respectively. While, the image enlargement in different ratio using scaling method is shown in Fig.3.(d), (e) and (f). Enlargement results in Fig.3.(d) and (e) show the salient content of image look a bit long, whereas Fig.3. (f) shows the
Akiyo rather meaty. Figures 3 (g), (h) and (i) show the image enlargement using our-method.

\subsection{Quantitative Evaluation}

We use quantitative evaluation using peak signal-to-noise ratio (PSNR) such as in Eq.(14).

$$
P S N R=10 \log \frac{M N \max (f(x, y))}{\sum_{x=1}^{M} \sum_{y=1}^{N}(f(x, y)-\hat{f}(x, y))^{2}}
$$


For this purpose, we create a scenario to test the image enlargement result using the PSNR analysis as follows: Firstly, we resize the standard image into the smaller size. The sizes of images which would be enlarged was $1 / 4$ of the standard image size. We use $t$ he nearest-neighborhood (NN) method to resize the standard image size. The next, we enlarge the small image size using all methods by the scale factor equal to four to become the standard image size or reference image size.

Figure 4 shows the PSNR result of the uniform image enlargement using scale factor equal to four. Our method has PSNR value higher than all comparison methods such as Bilinear Interpolation (BLI), Bicubic Interpolation (BCI), New Edge-Directed Interpolation (NEDI), Reverse Diffusion Interpolation (RDI), and Weighted Sum of Linear Extrapolations (WLE). This indicates our-method has good image enlargement results.

\section{Conclusions}

We have proposed the image enlargement method with different scale factor for the slice image region. The slice image region will be enlarged has a number of different columns. So different scale factor for width (SW) is implemented to obtain a full-size image enlargement. In this paper, the non-salient slice image region is selected manually. The proposed method has been evaluated using PSNR and resulting good image quality. The image enlargement in the different ratio has been presented.

For next study, we have plans to combine our method with feature detection to obtain auto-selection non-salient content in an image slice will be enlarged.

\section{Acknowledgment}

The author would like to thank the Directorate General of Higher Education Ministry of National Education (DGHE) of Indonesia wich providing scholarship.

\section{References}

(1) Xin Li, and Michael P. Orchard: "New Edge-Directed Interpolation", IEEE Trans. on Image Processing, vol 10, pp. 1521 - 1527, 2001.

(2) Olivier Salvado, Claudia M Hillenbrand and David L Wilson: "Partial Volume Reduction by Interpolation with Reverse Diffusion", International Journal of Biomedical Imaging, vol.2006, pp. 1 - 13, 2006.

(3) Takashi Aso, Noriaki Sutake and Takeshi Yamakau: "A Fast and Accurate Image Enlargement Algorithm Employing a Weighted Sum of Linear Extrapolations", Automation Congress, Proceedings. vol. 18, pp. 251 258, 2004.

(4) L.Q.Chen, X. Xie, X.Fan, W.Y.Man, H.J. Zhang and H.Q.Zhou: "A visual attention model for adapting image on small displays", Multimedia Syst., vol. 9 no. 4, pp.353-364, 2003.

(5) Y. Gou, F. Liu, J.Shi and Z.-H. ZZhou: "Image retargeting using mesh parametrization", IEEE Trans. on Multimedia, vol. 11, no. 5, pp.856-867,2009.

(6) W. Kim, and C. Kim: "A Texture-Aware Salient Edge Model for Image retargeting", IEEE Signal Processing Letterrs, vol. 18, no.11, pp.631-634, 2011.

(7) R. Achanta and S. Susstrunk: "Saliency detection for content-aware image resizing", in Proc. IEEE Int. COnf. Image Processing (ICIP), pp.1005-1008, 2009.

(8) S. Avidan and A. Shamir: "Seam carving for content-aware image resizing", ACM Trans. Graph. vol.26, no. 3, pp.267-276, 2007. 